\title{
Review \\ High-Tech Defense Industries: Developing Autonomous Intelligent Systems
}

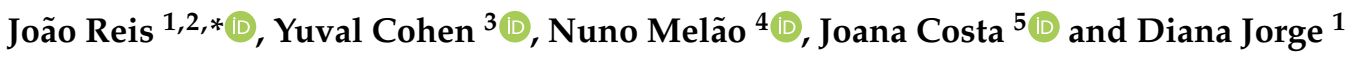 \\ 1 Industrial Engineering and Management, Faculty of Engineering, Lusófona University and EIGeS, \\ Campo Grande, 1749-024 Lisbon, Portugal; diana.jorge@ulusofona.pt \\ 2 Research and Development Center, Military University Institute, Rua de Pedrouços, \\ 1449-027 Lisbon, Portugal \\ 3 Department of Industrial Engineering, Afeka Tel-Aviv College of Engineering, Tel Aviv 69988, Israel; \\ yuvalc@afeka.ac.il \\ 4 CISeD-Research Center in Digital Services, Polytechnic Institute of Viseu, Campus Politécnico, \\ 3504-510 Viseu, Portugal; nmelao@estgv.ipv.pt \\ 5 Department of Economics, Management, Industrial Engineering and Tourism, GOVCOPP, Aveiro University, \\ Campus Universitário de Santiago, 3810-193 Aveiro, Portugal; joanacosta@ua.pt \\ * Correspondence: p40500@ulusofona.pt
}

check for updates

Citation: Reis, J.; Cohen, Y.; Melão, N.; Costa, J.; Jorge, D. High-Tech Defense Industries: Developing Autonomous Intelligent Systems. Appl. Sci. 2021, 11, 4920. https:// doi.org/10.3390/app11114920

Academic Editor: Andrea Prati

Received: 12 May 2021

Accepted: 26 May 2021

Published: 27 May 2021

Publisher's Note: MDPI stays neutral with regard to jurisdictional claims in published maps and institutional affiliations.

Copyright: (c) 2021 by the authors. Licensee MDPI, Basel, Switzerland. This article is an open access article distributed under the terms and conditions of the Creative Commons Attribution (CC BY) license (https:// creativecommons.org/licenses/by/ $4.0 /)$.

\begin{abstract}
After the Cold War, the defense industries found themselves at a crossroads. However, it seems that they are gaining new momentum, as new technologies such as robotics and artificial intelligence are enabling the development of autonomous, highly innovative and disruptive intelligent systems. Despite this new impetus, there are still doubts about where to invest limited financial resources to boost high-tech defense industries. In order to shed some light on the topic, we decided to conduct a systematic literature review by using the PRISMA protocol and content analysis. The results indicate that autonomous intelligent systems are being developed by the defense industry and categorized into three different modes-fully autonomous operations, partially autonomous operations, and smart autonomous decision-making. In addition, it is also important to note that, at a strategic level of war, there is limited room for automation given the need for human intervention. However, at the tactical level of war, there is a high probability of growth in industrial defense, since, at this level, structured decisions and complex analytical-cognitive tasks are carried out. In the light of carrying out those decisions and tasks, robotics and artificial intelligence can make a contribution far superior to that of human beings.
\end{abstract}

Keywords: artificial intelligence; defense industry; high technology; intelligent systems; level of war; robotics

\section{Introduction}

Advances in intelligent defense systems are growing and paving the way for high technological defense industries. Technologies such as robotics [1,2], artificial intelligence [3], and the internet of things [4] are driving considerable changes and impacts on defense industries [5]. These technologies are known to be capable of developing autonomous intelligent systems, increasingly designed for military applications and capable of operating efficiently in conflict areas and war zones. Thus, technology is often considered to be the key to many military revolutions in history, the so-called major military innovations or, as others have named, radical change in military affairs [6]. Three main factors are driving the latest revolution in military affairs: (1) rapid technological advancements that moved the Industrial Age into the Information Age; (2) the end of the Cold War; and (3) the decline in United States defense budgets [7].

In recent years, we have identified several studies on the development of autonomous defense systems in the industry sector. Some examples are presented in Zhang et al. [8], 
who focus on studying autonomous defense systems and relevant technological applications (e.g., X47-B, Predator, Global Hawk). However, apart from the division of warfare applications, to the best of our knowledge, no article has so far made a characterization of the autonomous defense systems literature in modes. That said, our intention is to explore and understand the following two research questions:

Research Question 1. How can the several modes of autonomous defense systems in the defense industry be categorized?

Research Question 2. How does the characterization of the autonomous defense systems modes contribute to making the defense industry highly technological?

Considering these research questions, it is quite evident that this research aims to understand and describe a real-life phenomenon, as suggested by Yin [9]. Although the research is of a qualitative and descriptive nature, it falls within the domain of applied sciences since it aims to identify the existing gaps and fill these gaps by initiating new scientific research in robotics and artificial intelligence applications at the various levels of war. It would seem, thus, inappropriate to carry out a more in-depth study to improve the high-tech defense industry without first knowing the holistic view of the use of autonomous defense systems.

This topic is important since there has been an exponential investment in autonomous intelligent systems in the military sector [5]. However, to the best of our knowledge, there has been no prior characterization of the modes of operations of these systems. In that regard, our article provides a comprehensive characterization of autonomous intelligent systems according to the various levels of war and different types of decisions and artificial intelligence. In addition to presenting a characterization of the various modes of autonomous intelligent systems in the defense industry, this article also characterizes how these modes contribute to making the defense industry highly technological. The novelty of this article is associated with the need to increase the degree of intelligent automation at the lowest levels of war. The need for automation is justified by the argument that, at the tactical/operational level, the military follows clear orders and makes structured decisions. Therefore, if structured decisions are made at the tactical level, which requires the performance of tasks of an analytical-cognitive nature, the type of intelligence needed is mechanical, thus, justifying the replacement of military personnel by machines, creating an excellent opportunity for the technological defense industries. On the other hand, at the strategic level of war, there is limited scope for the use of lethal autonomous weapon systems, given the need for human intervention, so as not to run into the current United Nations ethical, moral, and legal guidelines. Thus, this article's specific strengths are also associated with the managerial contribution, insofar as it provides the due knowledge on where to invest innovation and development resources of intelligent autonomous technologies, where they have a more significant growth perspective (i.e., in field operations-tactical level).

The remaining of the paper is organized as follows: the next section presents a conceptualization of the most relevant terms and applications; this is followed by an explanation of the methodological process; the results of this study are presented in Section 4, where the answers to the research questions can be found; in the last section, the conclusions are withdrawn, focusing on theoretical and managerial contributions, research gaps and suggestions for future work.

\section{Conceptual Background}

This section presents a brief conceptualization of the most relevant terms for this research. It also discusses the challenges for the defense industry and analyses the applications of autonomous intelligent systems in war.

\subsection{Concepts and Definitions}

In recent years, one of the most profound changes in the defense sector has been the use of robots, aiming to replace human beings in tasks of high precision and analytical-cognitive 
complexity [10], tasks of high risk for human life [11] and / or physically and physiologically strenuous [12,13]. To that end, the defense industry developed various sophisticated warfare applications that can operate in multiple domains (i.e., space, cyberspace, air, sea, and land). Due to a wide range of defense technologies, it is currently difficult, if virtually impossible, to describe consensual warfare applications among all defense researchers and academics. In addition to the growing demand for autonomous defense systems, a relevant argument that justifies the writing of this article is the degree of maturity that some military technologies have achieved [14]. The degree of maturity is somewhat related to the high learning curve of autonomous defense systems, due to the increased connectivity of these systems, via remote and network connection (e.g., fifth-generation technology standard for broadband cellular networks-5G) [15,16] and easy access to big data. Those characteristics allow autonomous defense systems to make decisions quickly, without empathetic or emotional impasses, which is an important distinctive characteristic when compared to human beings $[17,18]$. Thus, autonomous defense systems work without human interference, boosted by the latest advances in intelligence, cognition, computing, and system sciences [19]. When referring to autonomous defense systems, most are robots that detect, identify, understand, and interact autonomously with the external environment. Their capacity is based essentially on three functionalities: (1) sensors, which detect the environment characteristics [20]; (2) artificial intelligence, which identifies and understands the surrounding reality [21]; and (3) mechanisms, which allow real interaction [22].

Several articles in the literature carried out an analysis of automation, autonomy, and intelligence, which allows us to understand the most relevant terms. For instance, Insaurralde and Lane [23] define automation as the ability of a system to automatically carry out a certain process by following a code. They define autonomy as the ability of a system to carry out an automatic process by making decisions, implementing the choices made, and checking the evolution of such actions, i.e., make choices on its own. On the other hand, the human intelligence literature usually defines intelligence as the human ability to learn over time and adapt to the external environment [24,25]. Moreover, the artificial intelligence literature refers that intelligence is the capacity of mimicking human intelligence [26], such as the ability of knowledge and reasoning [27], problem-solving [28], communicating, interacting, and learning [29]. Huang and Rust [26] and Huang et al. [30] also proposed three artificial intelligences-mechanical, thinking, and feeling (Figure 1).

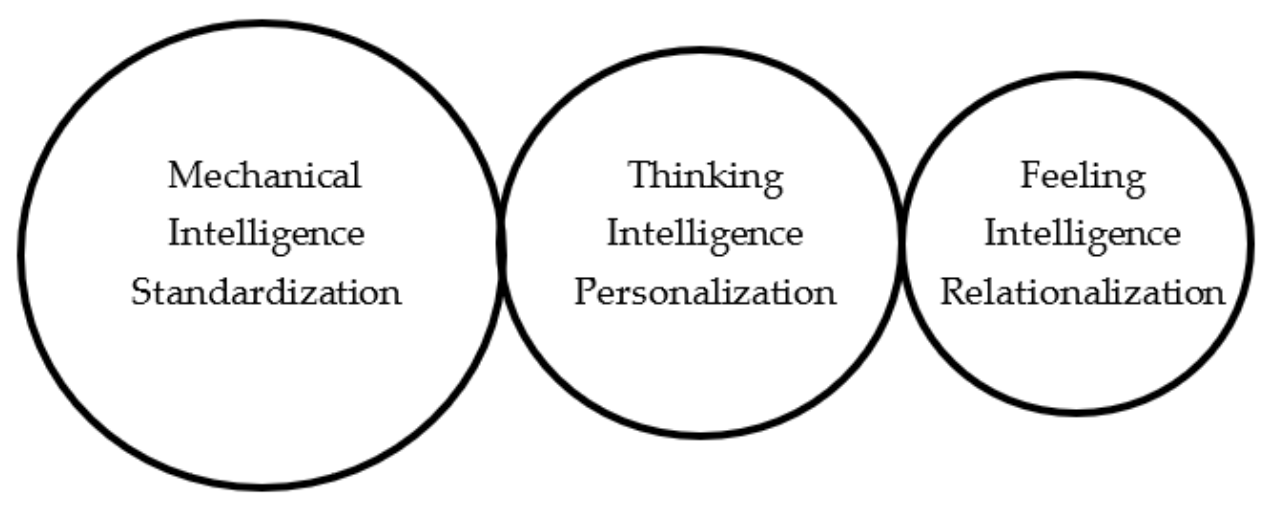

Figure 1. Three artificial intelligences (Adapted from Huang and Rust [26]).

Mechanical artificial intelligence is used for simple, standardized, repetitive, and routine tasks. An example of a civil application of mechanical intelligence is the use of service robots to clean hotel rooms, replacing humans in routine and standardized tasks [18]. Thinking artificial intelligence is used for complex, systematic, rule-based, and well-defined tasks. An example of this is Boston Dynamics' quadrupedal robots, highly adaptable, versatile, and capable of capturing the mobility, autonomy, and speed of living creatures [31,32]. Feeling artificial intelligence is used for social, emotional, communicative, 
and interactive tasks ([33], p. 32). Due to limited empathic and socioemotional capacity of machines, we believe that feeling artificial intelligence in the military domain should be performed by human beings. This argument is justified by the current state of social and emotional evolution of intelligent machines, which has not yet reached the desirable stage. For instance, it is difficult to imagine a lethal autonomous weapon system [34] in a combat situation with socioemotional ability capable of deciding whether to kill a child with a weapon in his possession. Such complex and socioemotional tasks require skills that are currently at the limit of the human being; some activities can eventually be shared with intelligent machines, but in the context of the decision-making process. We will hardly see a robot crying for a human in its current stage of evolution, whereas the opposite is already true. For example, when Boomer "died" in Iraq, the American soldiers offer him an impromptu military funeral-in this case Boomer was not a human being, but a robot whose job was to search for and defuse bombs [35]. Perhaps due to examples such as this one, robots have been increasingly integrated and accepted into military teams [36].

It is likely that the types of intelligence are also related to the types of decisions, as we will see in the Results section. Laudon and Laudon [37] classified decisions as structured, semi-structured, and unstructured. According to these authors, unstructured decisions are those in which decision-makers must evaluate and decide about something that is new, not routine, and for which there is no previously defined procedure. These decisions are made at the strategic level of the organization. In contrast, structured decisions are repetitive and routine, where established rules and accepted procedures are previously defined. Decisions of this type are taken at the operational management level. Intermediate decisions are semi-structured, where only part of the problem has a clear answer defined by a well-accepted procedure.

\subsection{Type and Levels of Autonomy}

The type and levels of autonomy are also well described in the literature, although the measurement scales are not always consensual, making it difficult to select a model. In that regard, Vagia et al. [38] presented a literature review of the evolution of the levels of autonomy since the end of 1950s (Table 1).

Table 1. A taxonomy for the levels of autonomy (Adapted from Vagia et al. [38]).

\begin{tabular}{ll}
\hline \multicolumn{1}{c}{ Levels of Automation } & \multicolumn{1}{c}{ Description } \\
\hline Level 1-Manual control. & $\begin{array}{l}\text { The computer offers no assistance. } \\
\text { The computer offers some decision to the operator. The operator } \\
\text { is responsible for deciding and executing. } \\
\text { Tevel 2-Decision proposal stage. }\end{array}$ \\
$\begin{array}{ll}\text { The human selects one decision, and the computer executes. } \\
\text { The computer selects one decision and executes with human } \\
\text { approval. }\end{array}$ \\
$\begin{array}{ll}\text { The computer executes the selected decision and informs the } \\
\text { hevel 5-Computer execution and on human information stage. }\end{array}$ \\
$\begin{array}{l}\text { Level 6-Computer execution and on-call human } \\
\text { information stage. }\end{array}$ & $\begin{array}{l}\text { The computer executes the selected decision and informs the } \\
\text { human only if asked. } \\
\text { The computer executes the selected decision and informs the } \\
\text { human only if it decides to. }\end{array}$ \\
The & $\begin{array}{l}\text { The computer does everything without human notification, } \\
\text { except if an error that is not into the specifications occurs. In } \\
\text { that case, the computer needs to inform the operator. }\end{array}$ \\
\hline
\end{tabular}

One of the most interesting aspects we highlight in Table 1 is the introduction of the failure mode at the fully autonomous control stage (level 8), which means the computer operates completely autonomously unless an unexpected error occurs. If the error does not appear in the program specifications, the computer must seek human support. This human support is relevant to the subject of this paper since, at the limit of the use of military technologies, there may be a need to attack human beings without any margin of error 
in selecting those who live or die. The intervention of human beings was not frequently considered at this level, as observed in one of the most relevant studies about levels of autonomy, published by Endsley and Kaber [39]. The taxonomy of Vagia et al. [38] intends to combine a series of levels from other articles that seem to have no significant differences between them, presenting the reader with a simpler and easier-to-understand model. The authors also intended to present a taxonomy that could be widely used, and, in this regard, the authors stated that it is up to the user to decide how to create their own taxonomy at the automation level and prioritize their needs and requirements. The modes of autonomous intelligent systems that we further developed show some similarities with the model of Vagia et al. [38], although it is not as comprehensive as it focuses on a specific domain (i.e., military field).

\subsection{Military Applications of Autonomous Intelligent Systems}

The defense industry is increasingly conducting autonomous defense systems research and development in several domains: space, cyberspace, air, sea, and land. It is in that regard that we present an overview of military application of autonomous defense systems in each of the aforementioned domains:

- Space robotics and autonomous intelligent systems;

- Autonomous intelligent cyber-defense agents;

- Intelligent unmanned autonomous systems - in the air, at sea, and on land.

Space robotics autonomous intelligent systems are presented as machines capable of operating in space and carrying out exploration in adverse environments that may not be found in the natural conditions of earth. In general, space robots are divided into two types: (1) orbital robots [40], which are characterized by robotics in low earth orbit or robotics in geostationary orbit; and (2) planetary robots [41], which are capable of closely examining extraterrestrial surfaces. As mentioned by Gao et al. [42], depending on the applications (orbital or planetary), space robots are often designed to be mobile, manipulate, grab, drill and/or collect samples, such as the National Aeronautics and Space Administration's recent Mars 2021 Perseverance Rover [43]. These robots are expected to have several levels of autonomy, from tele-operation by humans to fully autonomous operations. Depending on the level of autonomy, a space robot can act as [42]: (1) a robotic agent (or human proxy) that can perform tele-operation up to semi-autonomous operations; (2) a robotic assistant, who can help human astronauts that range from semi- to fully-autonomous operations; or (3) a robotic explorer capable of exploring unknown territory in space using fully autonomous operations. As an exemplary method, Giordano et al. [40] is presented as a validating benchmark. They reported an efficient fuel control strategy for a spacecraft equipped with a manipulator. Their key findings are associated with the strategy of using the thrusters, the reaction wheels, and the arm drives in a coordinated way to limit the use of the thrusters and achieve the ideal zero fuel consumption in non-contact maneuvers. The authors were able to validate the method via a hardware-in-the-loop simulator composed of a seven degrees of freedom arm mounted on a simulated six degrees of freedom spacecraft. Some military space robotics autonomous intelligent systems are related to spy satellites of some military powers and space-based missile defense systems.

Autonomous cyber defense is an area that has been driven by the defense sector in anticipation of threats to military infrastructures, systems, and operations [44]. These systems will be implemented through autonomous and intelligent cyber-defense agents that will fight against intelligent autonomous malware [44] and are likely to become primary cyber fighters on the future battlefield [45].

The popularity of advanced applications in the domains of air, land, and sea has also been steadily increasing [46] with the intelligent unmanned autonomous systems, which can perform operations without human intervention with the help of artificial intelligence [8]. In that regard, intelligent unmanned aircraft systems have aimed at autonomous flight, navigation, sensory, and decision-making capabilities [47] above conventional unmanned aircraft systems or unmanned aerial vehicles. Currently, unmanned 
aircraft systems and unmanned aerial vehicles systems have often been used for intelligence, surveillance, and reconnaissance activities or to carry out attack missions against high-value targets [48]. In addition, several methodologies have been targeted to these systems, such as Jourdan et al. [49], who designed an approach to mitigate common unmanned aerial vehicle failures, including primary control surface damage, airframe damage, and complete engine failure. Intelligent unmanned aircraft systems are beginning to be developed mainly in the civil context, such as package delivery [50], agriculture, and agroindustry [51], to name a few. The intelligent unmanned maritime and underwater systems have pushed the technology beyond imaginable limits in order to deal with complex ocean and sea missions [23]. These systems present new opportunities for naval use, in particular for dangerous missions, such as highly efficient mine sweeping. Recognizing the potential of autonomous underwater vehicles for both science and the military, in 1997 the Massachusetts Institute of Technology and the North Atlantic Treaty Organization joined efforts to develop robotic technologies applicable to mine countermeasures [52]. These systems have evolved with the use of disruptive technologies until today [53]. For example, Sands [54] developed very relevant methodologies in this regard. He studied deterministic artificial intelligence for unmanned underwater vehicles, proposing an automated control and learning methodology that requires simple user inputs. Noteworthy is also the attention that has been given to unmanned ground vehicles. Of these, an interesting example is the BigDog, developed by Boston Dynamics, which is a four-legged robot for transporting loads in difficult terrain [46] and has been adapted with autonomous navigation [55]. Moreover, mathematical structures and expectation-maximization and Gaussian mixture models algorithms have been developed [53]. Besides its applications in robotics and unmanned ground vehicles, these algorithms can also be used in various domains such as cybersecurity, object detection, and military logistics. The practical application of these algorithms has resulted in modern technology that can be applied on the battlefield, as presented by Bistron and Piotrowski [53], who give us examples of solutions such as Spot and Atlas (also developed by Boston Dynamics). In addition to the potential for civil society, much is speculated about the use of these autonomous robots in the revolution of military ground operations [6].

\section{Methodology}

This study was carried out as a systematic literature review, based on the original guidelines proposed by Moher et al. [56]. This research strategy is well justified by Fink [57], who argues that it is: systematic, since it embraces a methodological approach; explicit, as all data collection procedures are described in it; and comprehensive, as it brings together a wide range of scientific knowledge. The search process was undertaken in Elsevier's Scopus, one of the world's largest abstract and citation databases of peerreviewed literature. By choosing Scopus over other scientific databases (e.g., Web of Science, EBSCO Host, ScienceDirect) and/or academic search engines (e.g., Google Scholar), this article takes advantage of (1) greater transparency and easy replicability of results [58], (2) superior coverage of journals in the fields of applied and technological sciences [59], (3) peer-reviewed articles, which increases the quality of the results and the credibility of the research, (4) wider application of filters, and (5) immediate generation of search results in graphs and tables. Thus, based on the previous advantages, the use of a single scientific database has been accepted by the academic community [59]. By following the assumptions of Moher et al. [56], this research uses the PRISMA Statement (also known as Preferred Reporting Item for Systematic Reviews and Meta-Analysis), which consists of a 27-item checklist and a four-phase flow diagram. The checklist includes items considered essential for the transparent reporting of a systematic review [60]. As Page [61] points out, each item on the checklist was accompanied by an "explanation and elaboration", providing additional pedagogical justifications and guidance for each item, along with examples to demonstrate complete reports. The adoption of the guideline has been extensive, as indicated by its citation in tens of thousands of systematic reviews and frequent use as 
a tool to assess the integrity of published systematic review reports [62]. To do so, the search was initially conducted on 14 April 2021, by identifying documents with the terms "Defense Industry" and "Intelligent Systems" in all fields of search.

Following the typical application of filters during the screening phase of the PRISMA protocol, manuscripts were selected by source type, document type, and language (Table 2). The screening phase allowed more accurate results to be obtained. The selection of the source and type of document is closely related to the quality of the publication, as there seems to be a consensus among the academic community that journal articles are those that generally have superior scientific quality. Moreover, the option of selecting articles in English seemed to be an adequate option in order to avoid misinterpretations of the articles; on the other hand, as English is used worldwide, it involved a greater article coverage when compared to any other language. Following, the eligibility phase allowed to exclude articles that were not strictly related to the topic; and the inclusion criteria, which allowed to add manuscripts that were relevant to corroborate some information retrieved from Scopus. The final sample consisted of 62 articles from peer-reviewed journals (Table 2). The data analysis followed the technique known as content analysis $[63,64]$ in order to highlight new concepts and ideas [65]. The first phase started with the reading of all articles so as to identify similar words and terms [66]. After grouping the text, we identified the most relevant categories and subcategories [67]. In a next phase, we hierarchized the categories and subcategories to identify the patterns and ideas [68]. To assist in the analysis, we used a qualitative data analysis software-NVivo 11 [69], whose process allowed to obtain a holistic view of the existing literature, which is presented in the next chapter.

Table 2. Scopus search with PRISMA statement.

\begin{tabular}{lc}
\hline \multicolumn{1}{c}{ Elsevier's Scopus ${ }^{\circledR}$ Database } & $n$ \\
\hline Identification & \\
“Defense Industry” AND “Intelligent Systems” (All fields) & 153 \\
Screening & 67 \\
Source type (Journals) & 61 \\
Document type (Articles) & 57 \\
Language (English) & 51 \\
Eligibility & 52 \\
Full-text articles assessed for eligibility & 62 \\
Included & \\
\hline
\end{tabular}

\section{Results}

Since early, Hetrick [70] defined the high-tech defense in four manufacturing industries. The first two manufacturing industries are known as the aerospace industryaircrafts, guided missiles, and space vehicles; the third is the ordinance and accessories, and the fourth corresponds to navigation systems and equipment manufacturing. All of these industries can operate in the development of autonomous defense systems. One example is the research on ammunitions in future intelligent combat, which is a timely topic [71]. After carefully evaluating the applications of autonomous defense systems, it is safe to argue that three generic modes can be identified in the defense industry.

\section{Three Modes of Autonomous Intelligent Systems in the Defense Industry}

We identified high-technological autonomous defense systems that are being developed by the defense industry, and that can be categorized in three different modes (Table 3). The proposal to categorize autonomous defense systems modes is due to the scarcity of scientific production that favors a taxonomy for this type of systems, both autonomous and intelligent. Given the above, we continue by answering both research questions through real-life examples, taking into account the challenges and opportunities of each modality. 
Table 3. Modes of autonomous intelligent systems in the defense industry.

\begin{tabular}{|c|c|c|c|c|}
\hline $\begin{array}{l}\text { Modes of Autonomous } \\
\text { Intelligent Systems }\end{array}$ & Description & Levels of War & Decisions Type & $\begin{array}{l}\text { Types of Artificial } \\
\text { Intelligence }\end{array}$ \\
\hline $\begin{array}{l}\text { Mode 1-Fully } \\
\text { autonomous operation }\end{array}$ & $\begin{array}{l}\text { The human has no control over } \\
\text { the operation }\end{array}$ & $\begin{array}{l}\text { Military } \\
\text { tactical }\end{array}$ & Structured decision & Mechanical intelligence \\
\hline $\begin{array}{l}\text { Mode 2-Partially } \\
\text { autonomous operation }\end{array}$ & $\begin{array}{l}\text { The human has some kind of } \\
\text { control over the operation, or the } \\
\text { system assists humans and } \\
\text { vice-versa }\end{array}$ & $\begin{array}{l}\text { Military } \\
\text { operational }\end{array}$ & Semi-structured decisions & $\begin{array}{l}\text { Thinking } \\
\text { intelligence }\end{array}$ \\
\hline $\begin{array}{l}\text { Mode 3- Smart } \\
\text { autonomous } \\
\text { decision-making }\end{array}$ & $\begin{array}{l}\text { The intelligent system supports } \\
\text { humans in case of need }\end{array}$ & $\begin{array}{l}\text { Military } \\
\text { strategic }\end{array}$ & Unstructured decisions & $\begin{array}{l}\text { Feeling } \\
\text { intelligence }\end{array}$ \\
\hline
\end{tabular}

- Mode 1. Fully autonomous operation (the human has no control over the operation).

The doctrine of war is normally divided into three levels-strategic, operational, and tactical [72,73]. The first mode of autonomous defense systems usually operates at the tactical level, as it requires structured decisions in the field of operations. While the Mode 1 has greater efficiency when compared to humans, humans are expected to be replaced by machines in the middle and long term. Taking into account the previous argument, Mode 1 is expected to strongly contribute to make the defense industry highly technological. At lower levels of war (i.e., tactical level), structured decisions are made since soldiers are limited to following well-defined orders on the battlefield. In these situations, the performance of tasks is of an analytical-cognitive nature, where mechanical intelligence is the most recommended; that is, machines must take control of military operations. One example of Mode 1 from the data collected from Scopus is given by Rossiter [6], who recently published an article on the use of unmanned ground vehicles and how they are revolutionizing ground military operations. Rossiter [6] corroborates our previous arguments in the extant that unmanned ground vehicles' use has been intensified in recent years due to artificial intelligence, advanced computing, sensors, vision systems, and propulsion technologies. Rossiter [6] also refers that the United States Autonomous Systems Strategy (published in 2017) defined as a medium-term priority (2021-2030) the need to have fully autonomous unmanned combat vehicle operations. However, when it comes to the use of unmanned ground vehicles, recent history has shown that technological developments are promising but, so far, are unsatisfactory due to the created expectations. Thus, expectations have been made that there will be machines that will completely replace humans on the battlefield, but the defense industry is far from achieving that goal. In short, as can be seen in Table 3, fully autonomous operations (Mode 1) operate at the level of tactical warfare where structured decisions are made and which, in turn, refer to a type of mechanical intelligence. As far as mechanical intelligence is concerned, humans are likely to start losing control of these military operations to machines. Building upon the previous arguments, the next natural step is to define the construction elements of different types of weapons, as suggested by Božanić et al. [74], in order to produce autonomous systems suitable for users.

- Mode 2. Partially autonomous operation (the human has some kind of control over the operation, or the system assists humans and vice-versa).

Mode 2 is suitable for the operational level of warfare, as humans generally need to make semi-structured decisions with a medium level of responsibility. In this case, machines are recommended to take control over military operations, albeit with some kind of human control. An example of Mode 2 was also obtained from Scopus. In a first stage, it was possible to ascertain that in the context of the naval defense situation, awareness of the battle space is mission-critical. According to Dalkiran et al. [75], in platform-centered warfare, each combat unit keeps an individual situational awareness that is limited by a geographical area due to the limited range of the sensors (e.g., radar, optical and infrared sensors, sonar). The authors also refer that it is possible to achieve a shared battlefield awareness by connecting combat management systems on warships with command and 
control systems. Therefore, they proposed a communication mechanism for integrating data distribution service systems in real-time, where a combat management system could support military personnel on a warship with two main functions: (1) creating awareness of the battle space in real-time; (2) eliminating enemy forces using onboard weapon systems. According to Dalkiran et al.'s [75] research, a combat management system generates a tactical image with tracking data from various sensors and using data fusion algorithms. Subsequently, the collected information is sent to the weapon systems at high frequencies to engage enemy forces. These systems are generally used at an operational and strategic level, helping commanders to accomplish the security mission. Briefly, and as can be seen in Table 3 , military operations are partially autonomous. These are carried out at the operational level of war and where semi-structured decisions are made. These levels/decisions result in the domain of thinking intelligence, as mentioned by Huang and Rust [25,32]. This type of intelligence applies to complex tasks based on well-defined rules. Therefore, it is likely that humans will also lose some control of these military operations to the machines or to models which support decision making [76-78]. Typically, artificial intelligence algorithms are used to simulate the human analytical, cognitive, and intuitive components.

- Mode 3. Smart autonomous decision-making (the intelligent system supports human decision).

Mode 3 is suitable for strategic military operations, as humans are usually required to take complex and unstructured decisions with a high level of responsibility. In that regard, autonomous defense systems advise and support the human decision-making process, but do not replace humans in their decision. As these advanced systems are intelligent and autonomous, the upper limit of their military use can result in serious injury or loss of human life's, which means that, at a strategic level of war, it may be advisable to maintain some degree of human intervention and decision-making, preventing machines from killing humans indiscriminately in case of error. Furthermore, whereas in the manufacturing and services industry some degree of automation may be acceptable at high levels of decision, in the military and defense industry this is highly discouraged. So, it is necessary to find ways that allow the development of intelligent machines without coming up against the current ethical, moral, and legal guidelines of the United Nations (UN) [79,80]. These guidelines emphasize responsibility in deciding by humans over machines to safeguard interest in preserving human life. Given the above, it is likely that there will be little room for automation in Mode 3, which will not contribute to the same extent as Mode 1 to make the defense industry highly technological. From the content analysis, we can verify a relevant finding associated with the diversity of the degree of performance of the autonomous defense systems at the strategic level. That is, the autonomous defense systems that operate at the strategic level, mostly also operate at the lowest levels of the war. An example of the previous argument is unmanned aerial vehicles, which, due to their exponential growth and wide variety, can be used at all levels of war. The range of performance of unmanned aerial vehicles is discussed by Hamurcu and Eren [81] when they argue that they can be piloted remotely or fly autonomously or semi-autonomously. Their variety is mainly due to technological advances in robotics, which can imply widespread use, ranging from surveillance, tactical reconnaissance, and combat operations. If within the scope of surveillance and reconnaissance it can be acceptable for unmanned aerial vehicles to be autonomous and intelligent, with regard to combat operations, there has been much resistance in automating their decision.

Table 3 summarizes all the information regarding the autonomous defense systems Modes in the defense industry. Below we present the section of conclusions that focus on the main contributions of this research.

\section{Conclusions}

The purpose of this article was twofold. It first presented a characterization of the various modes of autonomous intelligent systems in the defense industry. Then, it characterized how those modes contribute to making the defense industry highly technological. 
The results indicate that the autonomous intelligent systems can be categorized in three different modes-fully autonomous operations, partially autonomous operations, and intelligent autonomous decision-making. With regard to Mode 1, since autonomous defense systems perform complex analytical-cognitive operations more efficiently than humans, it is likely that the latter will be replaced by machines in the medium and long term. Mode 2 is recommended to be run by autonomous defense systems, while humans can assume some kind of control. At this level, operational missions are developed, where human beings are called upon to make semi-structured decisions. Finally, at a strategic level, autonomous defense systems support humans in case of need. Therefore, in this specific case, autonomous defense systems only support but do not replace humans in its decision. Each mode contributes differently to making the defense industry highly technological. In other words, from our analysis, we conclude that it is likely that automation in Mode 3 will be much more limited when compared to Mode 1 . This is because in Mode 3 there must be a higher degree of decision and human intervention, which surpasses the previous modes that should have a higher degree of automation. These results provide guidance on the levels of automation where defense managers must invest their time and resources in research and development. So, scientific research in military technologies is recommended to focus on the first two levels of war (i.e., Tactical and Operational). As for the strategic level, the technological application is still very limited in the domain of empathic capacity in decision making, so it will likely continue to be operated in the hands of humans.

As this article follows a systematic literature review, some limitations are worth mentioning. First, the research presents a snapshot of the reality. Indeed, the Scopus database is constantly being updated, and, thus, it is likely that some relevant research has been left out of the analysis. Second, it is possible that other articles are left out of the analysis due to the application of filters. However, the PRISMA protocol allows the inclusion of relevant articles, mitigating the effects of that exclusion. Third, if different keywords are selected on Scopus, the search may also yield different results. However, after having performed several simulations with synonymous words, we found that the keywords chosen were those with the largest number of search results. Finally, the results have not been empirically tested and validated. Despite the aforementioned limitations, the analysis of the existing literature allowed the presentation of a holistic view of the phenomenon, which is focused on the autonomous defense systems in the context of defense, but also to provide managerial guidelines on where to invest the resources. Following this article, we suggest to empirically test and validate the findings to analyze if the research and development in the defense industry is moving in the right way. A second study can be focused on the technological transfer to the production industry and its commercialization, given that many of the existing theoretical studies end up not being carried out in practice. Finally, it may be interesting to study complementary fields of research for intelligent autonomous systems. This is the case of notable researchers such as Kwon et al. [82,83] who bring us neural network applications and adversarial examples. These military applications allow to deceive enemy classifiers and to safeguard the tactical use of intelligent autonomous systems.

Author Contributions: Conceptualization, J.R.; methodology, J.R.; software, J.R.; validation, Y.C., N.M., J.C. and D.J.; formal analysis, J.R.; investigation, J.R.; resources, J.R.; data curation, J.R.; writingoriginal draft preparation, J.R.; writing - review and editing, J.R., N.M. and D.J.; visualization, J.R.; supervision, N.M.; project administration, J.R.; funding acquisition, J.R. and Y.C. All authors have read and agreed to the published version of the manuscript.

Funding: This research received no external funding.

Institutional Review Board Statement: Not applicable.

Informed Consent Statement: Not applicable.

Data Availability Statement: The data used in this study was collected by the authors, any questions or clarifications can be addressed to the first author (p40500@ulusofona.pt). 
Acknowledgments: We would like to thank the contribution of the anonymous reviewers.

Conflicts of Interest: The authors declare no conflict of interest.

\section{References}

1. Lin, P.; Bekey, G.; Abney, K. Autonomous Military Robotics: Risk, Ethics, and Design. California Polytechnic State University of San Luis Obispo. 2008. Available online: https://apps.dtic.mil/sti/pdfs/ADA534697.pdf (accessed on 14 April 2021).

2. Ha, Q.; Yen, L.; Balaguer, C. Robotic autonomous systems for earthmoving in military applications. Autom. Constr. 2019, 107, 102934. [CrossRef]

3. Mori, S. US defense innovation and artificial intelligence. Asia-Pac. Rev. 2018, 25, 16-44. [CrossRef]

4. Mariani, J.; Williams, B.; Loubert, B. Continuing the March: The Past, Present, and Future of the IoT in the Military. The Internet of Things in Defense. Technical Report. 2015. Available online: https://www2.deloitte.com/us/en/insights/focus/internet-ofthings / iot-in-military-defense-industry.html (accessed on 14 April 2021).

5. Payal, M.; Dixit, P.; Sairam, T.; Goyal, N. Robotics, AI, and the IoT in Defense Systems. In AI and IoT-Based Intelligent Automation in Robotics; Wiley-Scrivener: Hoboken, NJ, USA, 2021.

6. Rossiter, A. Bots on the ground: An impending UGV revolution in military affairs? Small Wars Insur. 2020, 31, 851-873. [CrossRef]

7. Tilford, E. The Revolution in Military Affairs: Prospects and Cautions. US Army War College. 1995. Available online: https: //www.jstor.org/stable/pdf/resrep11803.pdf (accessed on 14 April 2021).

8. Zhang, T.; Li, Q.; Zhang, C.; Liang, H.; Li, P.; Wang, T.; Li, S.; Zhu, Y.; Wu, C. Current trends in the development of intelligent unmanned autonomous systems. Front. Inf. Technol. Electron. Eng. 2017, 18, 68-85. [CrossRef]

9. Yin, R. Case Study Research and Applications: Design and Methods; SAGE Publications: Thousand Oaks, CA, USA, 2018.

10. Chamola, V.; Kotesh, P.; Agarwal, A.; Gupta, N.; Guizani, M. A Comprehensive Review of Unmanned Aerial Vehicle Attacks and Neutralization Techniques. Ad. Hoc. Netw. 2020, 111, 102324. [CrossRef] [PubMed]

11. Šipoš, D.; Gleich, D. A lightweight and low-power UAV-borne ground penetrating radar design for landmine detection. Sensors 2020, 20, 2234. [CrossRef] [PubMed]

12. Sapaty, P. Military robotics: Latest trends and spatial grasp solutions. Int. J. Adv. Res. Artif. Intell. 2015, 4, 9-18.

13. Walsh, G.; Low, D. Military load carriage effects on the gait of military personnel: A systematic review. Appl. Ergon. 2021, 93, 103376. [CrossRef]

14. Rossiter, A. The impact of robotics and autonomous systems (RAS) across the conflict spectrum. Small Wars Insur. 2020, 31, 691-700. [CrossRef]

15. Garg, S.; Aujla, G.; Erbad, A.; Rodrigues, J.; Chen, M.; Wang, X. Guest Editorial: Blockchain Envisioned Drones: Realizing 5G-Enabled Flying Automation. IEEE Netw. 2021, 35, 16-19. [CrossRef]

16. Rathje, J.; Katila, R. Enabling technologies and the role of private firms: A machine learning matching analysis. Strategy Sci. 2021, 6, 5-21. [CrossRef]

17. Reis, J.; Melão, N.; Salvadorinho, J.; Soares, B.; Rosete, A. Service robots in the hospitality industry: The case of Hennna hotel, Japan. Technol. Soc. 2020, 63, 101423. [CrossRef]

18. Rosete, A.; Soares, B.; Salvadorinho, J.; Reis, J.; Amorim, M. Service robots in the hospitality industry: An exploratory literature review. In International Conference on Exploring Services Science; Springer: Cham, Switzerland; Porto, Portugal, 2020; pp. 174-186.

19. Wang, Y.; Hou, M.; Plataniotis, K.; Kwong, S.; Leung, H.; Tunstel, E.; Rudas, I.; Trajkovic, L. Towards a theoretical framework of autonomous systems underpinned by intelligence and systems sciences. IEEE/Caa J. Autom. Sin. 2020, 8, 52-63.

20. Campbell, S.; O'Mahony, N.; Krpalcova, L.; Riordan, D.; Walsh, J.; Murphy, A.; Ryan, C. Sensor technology in autonomous vehicles: A review. In Proceedings of the 2018 29th Irish Signals and Systems Conference (ISSC), Belfast, UK, 21-22 June 2018; pp. $1-4$.

21. Horowitz, M.C.; Scharre, P.; Velez-Green, A. A Stable Nuclear Future? The Impact of Autonomous Systems and Artificial Intelligence. arXiv 2019, arXiv:1912.05291. Available online: https:/ /arxiv.org/abs/1912.05291 (accessed on 20 April 2021).

22. Sandin, P. Robot Mechanisms and Mechanical Devices Illustrated; McGraw Hill Professional: New York, NY, USA, 2003.

23. Insaurralde, C.; Lane, D. Metric assessment of autonomous capabilities in unmanned maritime vehicles. Eng. Appl. Artif. Intell. 2014, 30, 41-48. [CrossRef]

24. Schlinger, H. The Myth of Intelligence. Psychol. Rec. 2003, 53, 15-32.

25. Sternberg, R. The Theory of Successful Intelligence. Interam. J. Psychol. 2005, 39, 189-202.

26. Huang, M.; Rust, R. Artificial intelligence in service. J. Serv. Res. 2018, 21, 155-172. [CrossRef]

27. Leidner, D. Cognitive Reasoning for Compliant Robot Manipulation; Springer International Publishing: Cham, Switzerland, 2019.

28. Ibarz, J.; Tan, J.; Finn, C.; Kalakrishnan, M.; Pastor, P.; Levine, S. How to train your robot with deep reinforcement learning: Lessons we have learned. Int. J. Robot. Res. 2021, 40, 0278364920987859. [CrossRef]

29. Richards, L.; Matuszek, C. Learning to Understand Non-Categorical Physical Language for Human Robot Interactions. UMBC Student Collection 2016. Available online: http:/ / hdl.handle.net/11603/21316 (accessed on 30 April 2021).

30. Huang, M.; Rust, R.; Maksimovic, V. The feeling economy: Managing in the next generation of artificial intelligence (AI). Calif. Manag. Rev. 2019, 61, 43-65. [CrossRef]

31. Raibert, M.; Blankespoor, K.; Nelson, G.; Playter, R. Bigdog, the rough-terrain quadruped robot. IFAC Proc. Vol. 2008, 41, 10822-10825. [CrossRef] 
32. Murphy, M.; Saunders, A.; Moreira, C.; Rizzi, A.; Raibert, M. The littledog robot. Int. J. Robot. Res. 2011, 30, 145-149. [CrossRef]

33. Huang, M.; Rust, R. Engaged to a robot? The role of AI in service. J. Serv. Res. 2021, 24, 30-41. [CrossRef]

34. Taylor, I. Who Is Responsible for Killer Robots? Autonomous Weapons, Group Agency, and the Military-Industrial Complex. J. Appl. Philos. 2020, 38, 320-334. [CrossRef]

35. Nyholm, S.; Smids, J. Can a robot be a good colleague? Sci. Eng. Ethics 2020, 26, 2169-2188. [CrossRef] [PubMed]

36. Bellas, A.; Perrin, S.; Malone, B.; Rogers, K.; Lucas, G.; Phillips, E.; Tossel, C.; Visser, E. Rapport building with social robots as a method for improving mission debriefing in human-robot teams. In Proceedings of the 2020 Systems and Information Engineering Design Symposium (SIEDS), Charlottesville, VA, USA, 24 April 2020; pp. 160-163.

37. Laudon, K.; Laudon, J. Management Information Systems; Pearson: Upper Saddle River, NJ, USA, 2015.

38. Vagia, M.; Transeth, A.; Fjerdingen, S. A literature review on the levels of automation during the years. What are the different taxonomies that have been proposed? Appl. Ergon. 2016, 53, 190-202. [CrossRef] [PubMed]

39. Endsley, M.; Kaber, D. Level of automation effects on performance, situation awareness and workload in a dynamic control task. Ergonomics 1999, 42, 462-492. [CrossRef]

40. Giordano, A.; Dietrich, A.; Ott, C.; Albu-Schäffer, A. Coordination of thrusters, reaction wheels, and arm in orbital robots. Robot. Auton. Syst. 2020, 131, 103564. [CrossRef]

41. Sancho-Pradel, D.; Gao, Y. A survey on terrain assessment techniques for autonomous operation of planetary robots. JBIS-J. Br. Interplanet. Soc. 2010, 63, 206-217.

42. Gao, Y.; Jones, D.; Ward, R.; Allouis, E.; Kisdi, A. Space Robotics and Autonomous Systems: Widening the Horizon of Space Exploration. UK-RAS White Paper, 2016. Available online: https://www.surrey.ac.uk/sites/default/files/UK_RAS_wp_print_ single_pages.pdf (accessed on 23 April 2021).

43. Voosen, P. Perseverance will explore history of ancient lake. Science 2021, 371, 870-871. [CrossRef]

44. Théron, P.; Kott, A. When Autonomous Intelligent Goodware Will Fight Autonomous Intelligent Malware: A Possible Future of Cyber Defense. In Proceedings of the MILCOM 2019-2019 IEEE Military Communications Conference (MILCOM), Norfolk, VA, USA, 12-14 November 2019; pp. 1-7.

45. Kott, A. Intelligent autonomous agents are key to cyber defense of the future army networks. Cyber Def. Rev. 2018, 3, 57-70.

46. Campbell, S.; Naeem, W.; Irwin, G. A review on improving the autonomy of unmanned surface vehicles through intelligent collision avoidance manoeuvres. Annu. Rev. Control 2012, 36, 267-283. [CrossRef]

47. Santoso, F.; Garratt, M.A.; Anavatti, S. State-of-the-art intelligent flight control systems in unmanned aerial vehicles. IEEE Trans. Autom. Sci. Eng. 2017, 15, 613-627. [CrossRef]

48. Udeanu, G.; Dobrescu, A.; Oltean, M. Unmanned aerial vehicle in military operations. Sci. Res. Educ. Air Force 2016, 18, 199-206. [CrossRef]

49. Jourdan, D.; Piedmonte, M.; Gavrilets, V.; Vos, D.; McCormick, J. Enhancing UAV survivability through damage tolerant control. In Proceedings of the AIAA Guidance, Navigation, and Control Conference, Toronto, ON, Canada, 2-5 August 2010 ; p. 7548.

50. Stolaroff, J.; Samaras, C.; O’Neill, E.; Lubers, A.; Mitchell, A.; Ceperley, D. Energy use and life cycle greenhouse gas emissions of drones for commercial package delivery. Nat. Commun. 2018, 9, 1-13.

51. Hartanto, R.; Arkeman, Y.; Hermadi, I.; Sjaf, S.; Kleinke, M. Intelligent Unmanned Aerial Vehicle for Agriculture and Agroindustry. In IOP Conference Series: Earth and Environmental Science; IOP Publishing: Bristol, UK, 2019; Volume 335, p. 012001.

52. Bovio, E.; Cecchi, D.; Baralli, F. Autonomous underwater vehicles for scientific and naval operations. Annu. Rev. Control 2006, 30, 117-130. [CrossRef]

53. Bistron, M.; Piotrowski, Z. Artificial Intelligence Applications in Military Systems and Their Influence on Sense of Security of Citizens. Electronics 2021, 10, 871. [CrossRef]

54. Sands, T. Development of Deterministic Artificial Intelligence for Unmanned Underwater Vehicles (UUV). J. Mar. Sci. Eng. 2020, 8, 578. [CrossRef]

55. Wooden, D.; Malchano, M.; Blankespoor, K.; Howardy, A.; Rizzi, A.; Raibert, M. Autonomous navigation for BigDog. In Proceedings of the 2010 IEEE International Conference on Robotics and Automation (ICRA), Anchorage, AK, USA, 3-7 May 2010; pp. 4736-4741.

56. Moher, D.; Liberati, A.; Tetzlaff, J.; Altman, D.; Prisma Group. Preferred reporting items for systematic reviews and meta-analyses: The PRISMA statement. PLoS Med. 2009, 6, e1000097. [CrossRef] [PubMed]

57. Fink, A. Conducting Research Literature Reviews: From the Internet to Paper, 2nd ed.; Sage Publications: Thousand Oaks, CA, USA, 2005.

58. Tranfield, D.; Denyer, D.; Smart, P. Towards a methodology for developing Evidence-Informed Management Knowledge by Means of Systematic Review. Br. J. Manag. 2003, 14, 207-222. [CrossRef]

59. Reis, J.; Santo, P.; Melão, N. Influence of artificial intelligence on public employment and its impact on politics: A systematic literature review. Braz. J. Oper. Prod. Manag. 2021, 18, 1-22. [CrossRef]

60. Liberati, A.; Altman, D.; Tetzlaff, J.; Mulrow, C.; Gøtzsche, P.; Ioannidis, J.; Clarke, M.; Devereaux, P.; Kleijnen, J.; Moher, D. The PRISMA statement for reporting systematic reviews and meta-analyses of studies that evaluate health care interventions: Explanation and elaboration. J. Clin. Epidemiol. 2009, 62, e1-e34. [CrossRef] [PubMed]

61. Page, M.; McKenzie, J.; Bossuyt, P.; Boutron, I.; Hoffmann, T.; Mulrow, C.; Shamseer, L.; Telzlaff, J.; Moher, D. Updating guidance for reporting systematic reviews: Development of the PRISMA 2020 statement. J. Clin. Epidemiol. 2021, 134, 103-112. [CrossRef] 
62. Page, M.J.; Moher, D. Evaluations of the uptake and impact of the Preferred Reporting Items for Systematic reviews and Meta-Analyses (PRISMA) Statement and extensions: A scoping review. Syst. Rev. 2017, 6, 1-14. [CrossRef]

63. Flick, U.; Kardorff, E.; Steinke, I. A Companion to Qualitative Research; Sage Publications: Thousand Oaks, CA, USA, 2004.

64. Neuendorf, K.; Kumar, A. Content analysis: An Introduction to Its Methodology. In The International Encyclopedia of Political Communication; Wiley Online Library: Cleveland, OH, USA, 2015; pp. 1-10. [CrossRef]

65. Krippendorff, K. Content Analysis: An Introduction to Its Methodology; Sage Publications: Thousand Oaks, CA, USA, 2018.

66. Stemler, S. An overview of content analysis. Pract. Assess. Res. Eval. 2000, 7, 17.

67. Elo, S.; Kyngäs, H. The qualitative content analysis process. J. Adv. Nurs. 2008, 62, 107-115. [CrossRef]

68. Hsieh, H.; Shannon, S. Three approaches to qualitative content analysis. Qual. Health Res. 2005, 15, 1277-1288. [CrossRef]

69. Bazeley, P.; Jackson, K. Qualitative Data Analysis with NVivo; Sage Publications: Thousand Oaks, CA, USA, 2013.

70. Hetrick, R. Employment in high-tech defense industries in a post-cold war era. Mon. Labor Rev. 1996, $119,57$.

71. Liu, D.; Sun, J.; Huang, D.; Wang, X.; Cheng, K.; Yang, W.; Ding, J. Research on development status and technology trend of intelligent autonomous ammunition. In Journal of Physics: Conference Series; IOP Publishing: Bristol, UK, 2021; Volume 1721, p. 012032.

72. MacGregor, D. Future Battle: The Merging Levels of War; Army War Coll Carlisle Barracks PA. 1992. Available online: https: / /apps.dtic.mil/sti/pdfs/ADA528099.pdf (accessed on 1 May 2021).

73. Kiszely, J. Thinking about the operational level. RUSI J. 2005, 150, 38-43. [CrossRef]

74. Božanić, D.; Ranđelović, A.; Radovanović, M.; Tešić, D. a hybrid lbwa-ir-mairca multi-criteria decision-making model for determination of constructive elements of weapons. Facta Univ. Ser. Mech. Eng. 2020, 18, 399-418.

75. Dalkıran, E.; Önel, T.; Topçu, O.; Demir, K. Automated integration of real-time and non-real-time defense systems. Def. Technol. 2021, 17, 657-670. [CrossRef]

76. Božanić, D.; Tešić, D.; Kočić, J. Multi-criteria FUCOM-Fuzzy MABAC model for the selection of location for construction of single-span bailey bridge. Decis. Mak. Appl. Manag. Eng. 2019, 2, 132-146. [CrossRef]

77. Malbašić, S.; Đurić, S. Risk assessment framework: Application of Bayesian Belief Networks in an ammunition delaboration project. Vojnoteh. Glas. 2019, 67, 614-641. [CrossRef]

78. Božanić, D.; Tešić, D.; Milić, A. Multicriteria decision making model with Z-numbers based on FUCOM and MABAC model. Decis. Mak. Appl. Manag. Eng. 2020, 3, 19-36. [CrossRef]

79. Arkin, R. Lethal autonomous systems and the plight of the non-combatant. In The Political Economy of Robots; Palgrave Macmillan: Cham, Switzerland, 2018; pp. 317-326.

80. Righetti, L.; Pham, Q.; Madhavan, R.; Chatila, R. Lethal autonomous weapon systems [ethical, legal, and societal issues]. IEEE Robot. Autom. Mag. 2018, 25, 123-126. [CrossRef]

81. Hamurcu, M.; Eren, T. Selection of Unmanned Aerial Vehicles by Using Multicriteria Decision-Making for Defence. J. Math. 2020. [CrossRef]

82. Kwon, H.; Yoon, H.; Choi, D. Restricted evasion attack: Generation of restricted-area adversarial example. IEEE Access 2019, 7, 60908-60919. [CrossRef]

83. Kwon, H.; Yoon, H.; Park, K. Acoustic-decoy: Detection of adversarial examples through audio modification on speech recognition system. Neurocomputing 2020, 417, 357-370. [CrossRef] 